\title{
ASSESSING THE IMPACTS OF CLIMATIC AND TELECONNECTION FACTORS ON MAIZE (ZEA MAYS L.) YIELD FROM BOTH MACROSCOPIC AND MICROSCOPIC PERSPECTIVES
}

\author{
LI, P. - HUANG, S. Z. - HUANG, Q. \\ State Key Laboratory of Eco-hydraulics in Northwest Arid Regionof China, Xi'an University of \\ Technology, Xi'an 710048, China \\ (phone: +86-29-8231-2801; fax: +86-29-8231-2797) \\ ${ }^{*}$ Corresponding author \\ e-mail: huangshengzhi7788@126.com; phone: +86-29-8231-2801; fax:+86-29-8231-2797
}

(Received 27 $7^{\text {th }}$ Sep 2019; accepted $4^{\text {th }}$ Feb 2020)

\begin{abstract}
Maize (Zea mays L) as an important crop in the world is strongly affected by climatic and teleconnection factors, it is therefore necessary to fully assess their impacts on maize yield. Given the complexity and non-stationarity of the maize yield fluctuations in China, the empirical mode decomposition (EMD) and cross-wavelet methods were adopted to explore the correlations between maize yield including its whole series and various frequency series decomposed by the EMD and climatic and teleconnection factors from both macroscopic and microscopic perspectives. Results indicated that: (1) from the macroscopic perspective, sunspots were the main influencing factor of maize yield; (2) from the microscopic perspective, mainly precipitation, temperature, El Niño-Southern Oscillation (ENSO), Atlantic Oscillation (AO), and Pacific Decadal Oscillation (PDO) impact the high-medium frequency components (i.e., the frequency domain is $1 \sim 4$ years and $6 \sim 10$ years) of the detrended maize yield sequence, whilst solar activities (i.e., sunspots) primarily influence its low-medium frequency components (i.e., the frequency domain is $8 \sim 14$ years); (3) three complete influencing hierarchical frameworks of sunspots-teleconnection factors-climatic factors on the various frequency components of the detrended maize yield were constructed, which help develop a physical significance of maize yield prediction model The study provides new insights into crop yield forecasting, thereby laying a solid scientific basis for crop yield decisions.
\end{abstract}

Keywords: EMD, the cross wavelet method, climate change, different perspectives

\section{Introduction}

Climate change has been one of the most studied topics during the last decades due to its socioeconomic, environmental and biological implications (IPCC, 2014; Fang et al., 2019; Guo et al., 2020). In the coming decades, global climate change will affect all sectors of the global economy (Huang et al., 2020; Ren et al., 2020; Li et al., 2020). But most of the impacts will concentrate on the agricultural sector, inducing food insecurity, particularly in developing countries (Ringler, 2008; Nelson et al., 2009). Despite significant advances in technology and in crop yield potential, food production and food security remain highly dependent on weather and climate change, as solar radiation, temperature and precipitation are the main drivers of crop growth (Angstrom, 1924; Hunt et al., 1998; Rosenzweig et al., 2001). Climate is one of the major uncontrollable factors affecting crop yield and causing global yield stagnation among the ones (You et al., 2009; Godfray et al., 2010). Maize is the crop with the largest planting area and production in China. According to FAO, the planted area of maize in 2016 was 36.8 million ha, and production was 219.6 million tons (Planting Industry Management Department, 2018; Tian et al., 2019; He et al., 2019). Therefore, it is necessary to explore the impact of climate change on corn yield to ensure the security of China's grain yield. 
Climate change is causing significant change in water supply and further threats to food production in various parts of the world (Hanjra et al., 2010; Knox et al., 2012; Smith et al., 2013; Zhao et al., 2013; Zhao et al., 2020a). As a result, food security problems caused by extreme climate events have sparked research and public interest in the analysis of climate change and agricultural production (Kummu et al., 2012). The increased rainfall recorded in the Pampas region led to yield increases of rainfed crops in the order of $38 \%$ for soybean, $18 \%$ maize, $13 \%$ wheat and $12 \%$ in sunflower (Zhang et al., 2008). The fluctuation of temperature has an adverse effect on food production (Magrin et al., 2005; Lobell et al., 2008; Battisti et al., 2009). ENSO condition has been related to agricultural yields in many parts of the world (Garnett et al., 1992; Cane et al., 1994; Hammer et al., 2001; Butler et al., 2013). Low maize yields in Zimbabwe were found to be a result of El Nino (Podestá et al., 2002; Butler et al., 2013). Similar negative effects caused by El Nino were also reported concerning lowland rice yields and total rice production during the wet season in the Philippines and Indonesia (Phillips et al., 1998; Lansigan et al., 2000). There was a significant negative correlation between the Atlantic oscillation (AO) index and maize and rice yield in northeast China (Naylor et al., 2001). Some complex impacts of the Pacific Decadal Oscillation (PDO) on dryland corn and wheat yields in the Missouri River Basin were found (Kim et al., 2005). Besides, solar radiation affects crop yield by affecting the process of crop growth (Angstrom et al., 1924; Hunt et al., 1998; Mehta et al., 2012), the change of crop yield is therefore affected to some extent by the intensity of solar activities (usually expressed by the number of sunspots). As mentioned above, long-term fluctuations in crop yield are closely related with climatic factors such as temperature and precipitation, teleconnection factors (i.e. ENSO, AO, PDO) (for the sake of brevity, climatic and teleconnection factors are collectively referred to as signals of climate variability) (Zhao et al., 2015a; Xu et al., 2018) and solar activities (i.e. sunspots). And, the above-mentioned research indicates that global climate change is slowly affecting the spatial pattern of global and regional food production and their yield levels, which plays an important role in investigating the food production changes and their possible causes under the context of global climate change. Nevertheless, these studies only considered the impact of a single factor on crop yield from a macroscopic perspective, neither comprehensively taking into account the combined effects of these factors on crop yield, nor exploring the differences of effects of the above-mentioned influencing factors on food production at different time scales including the macroscopic and microcosmic scales.

As previous studies mentioned that crop yield is affected by the signals of climate variability and solar activities. However, all of these factors possess different periodic signals. For instance, Plaut et al. (1995) reported the detection of 5.2, 7.7, 14.2 and 25.0 years periods in the 335 years the Central England Temperature by using the SSA technique. Chen et al. (2016) found that four significant cycles with alternation patterns of precipitation were detected mainly at the time scales of 3-5, 10-11, 20-23, and 31.2 years for each of the four subregions of Liaoning province. ENSO, first described in 1923 by Sir Gilbert Walker (Walker, 1923), is a coupled atmospheric-oceanic oscillation in the tropical Pacific with an average period of 2-7 years. Like ENSO, the PDO is dominated by oceanic temperature oscillations with a typical period of 20-30 years (Mantua et al., 1997; Zhang et al., 1997; Nigam et al., 1999; Stoner et al., 2009). The Arctic Oscillation is a mode of the climate variability with decadal-scale variability oscillation (Mokhov et al., 2013). Besides, average period of a sunspot number cycle is 11.2 years, actual periods have ranged from 7 to 14 years (Kane et al., 2008). In other words, the crop field under the influence of multiple signals of climate variability and solar activities must be provided with complex periodic 
signals. Therefore, studying the impact of the signals of climate variability and solar activities on crop yield from a macroscopic scale can only reveal the response characteristics of crop yield change to the changing environment in part.

In order to increase our awareness of the impacts of climate change and develop adaptation projects, it is necessary to separate the effects of climate change from the effects of each climate variable on observed changes in crop yields (Bai et al., 2015). Now that crop yield is the result of a combination of factors. Under the influence of multiple factors, its fluctuations do not move in a fixed cycle, but include changes in various time scales (periods) and local fluctuations, which lead it to change in multiple levels of time scales and localizations in the time domain. Hence, if the signal of which cycle of grain yield is affected specifically by the above factors can be explained clearly (that is, the effects of signals of climate variability and solar activities on different frequencies of maize yield are studied from the microcosmic scale), which will provide a very favorable forecasting approach for crop yield prediction, helping policy makers to accurately control the future trend of crop yield, and contributing to the reasonable development of national food security. The empirical mode decomposition (EMD) method is used to linearize and smooth the nonlinear and non-stationary signals step by step (Huang et al., 1998, 2013; Zhao et al., 2017; Yu et al., 2018; Zhao et al., 2020b). The method can separate the fluctuations of different scales step by step, and produce a series of intrinsic mode functions (IMFs) containing local characteristic information at different time scales of the original signal, and the residue $(\mathrm{R})$, which retains the data itself in the process of decomposition characteristic. Therefore, this study will employ the EMD method for wave decomposition analysis, with a purpose of exploring the multi-scale characteristics of China's maize yield fluctuations and their correlations with major influencing factors (including the signals of climate variability and solar activities), which provide a new insight into the impact of various influencing factors on maize yield fluctuations at multiple scales including the macroscopic and microcosmic scales. This work helps to physically reveal the impact of climate change on grain production variations, thereby being helpful for reliable grain production forecasting. Additionally, since overall trend in increasing food production mainly caused by human activities (e.g. technological advances, fertilization, irrigation, etc.) (Lu et al., 2017), the yield data employed in this study is detrended in order to focus on analyzing climate effect on crop yield.

The main objectives of this study therefore are: (1) to examine the changing periods of detrended maize yield and the IMFs of maize yield in China; (2) to explore the possible causes of detrended maize yield variation from the perspective of climate change including the signals of climate variability (i.e. precipitation, temperature, AO, ENSO, PDO) and solar activities (i.e. sunspots) at the macroscopic scale; (3) to investigate the related implications of the signals of climate variability and sunspots on maize yield decompositions at the microcosmic scale; (4) to construct a complete influencing hierarchical framework of sunspots-teleconnection factors (ENSO, AO, PDO)-climatic factors (precipitation and temperature) on the various frequency components of the detrended maize yield. The remainder of the paper is structured in four sections. Section 2 describes the study database and methods. Results and discussion are provided in Section 3 and Section 4, Section 5 presents the conclusions. 


\section{Data sources and methodology}

\section{Data sources}

This study used annual, country-level yield data for corn during 1961-2016 from Food and Agriculture Organization of the United Nations (http://www.fao.org/faostat/en/\#home). The meteorological data (included, precipitation, temperature) from the China meteorological data network (http://data.cma.cn/). The ENSO indices were derived from the National Oceanic and Atmospheric Administration (NOAA) Earth System Research Laboratory (www.esrl.noaa.go -v/psd/data/correlation/amon.us.data) and www.esrl.noaa.gov/psd/data/correlation/nina34.data), the AO index was derived from the NOAA National Climatic Data Center (www.ncdc.noaa.gov/tele -connections/ao/), and the PDO index was derived from the Tokyo Climate Center (ds.data.jma.go.jp/tcc/tcc/products/elnino/decadal/annpdo.txt).

\section{Methods}

\section{The Empirical Mode Decomposition}

Empirical Mode Decomposition (EMD) is an adaptive method for separating the spectrum of nonlinear and non-stationary signals (Wu et al., 2007). It decomposes a given time series, or signal, in components with different frequencies and amplitudes, known as Intrinsic Mode Functions (IMFs). IMFs have two attributes that differentiate them from other signals:

- The number of extreme and zero crossings must differ at most by one.

- The mean value between the upper and lower envelope is zero.

The original time series $X(\mathrm{t})$ can be written as Eq.l.

$$
X(\mathrm{t})=\sum_{i=1}^{m} I M F_{i}(\mathrm{t})+\mathrm{r}(\mathrm{t})
$$

where $m$ is the number of IMFs.

The Stopping Criterion for the extraction of each IMF consists of verifying whether or not the component $\mathrm{h}$ can be defined as an IMF, as well as optional criterion such as the component maintaining its characteristics after $S$ additional number of siftings (S-number) and a maximum number of siftings. The sifting process runs iteratively, extracting IMFs from the signal until the residue becomes a monotonic function, a constant value or a function with only one extremum from which no more IMFs can be extracted (Zhao et al., 2017).

\section{The cross wavelet analysis}

The cross wavelet analysis developed by Hudgins et al. (1993) is a new technique in exploring the associations between two associated time series. It combines wavelet transform with cross-spectrum analysis to ideally reflect the variation characteristics and coupled oscillations of the two time series in the time and frequency fields. (Hudgins et al., 1993; Torrence et al., 1998).

The cross wavelet transform of two time series $x_{n}$ and $y_{n}$ is expressed as $W^{X Y}=W^{X} W^{Y^{*}}$ where $*$ denotes their complex conjugation. The cross wavelet power can be expressed as $\left|W^{X Y}\right|$. The complex argument $\arg \left(W^{X Y}\right)$ can be regarded as the local relative phase 
between $x_{n}$ and $y_{n}$ in time-frequency field. The theoretical distribution of the cross wavelet power of these two series with their background power spectra $P_{K}^{X}$ and $P_{K}^{Y}$ is expressed as follows (Hudgins et al., 1993):

$$
D\left(\frac{W_{n}^{X}(S) W_{n}^{Y^{*}}}{\sigma_{x} \sigma_{y}}<p\right)=\frac{Z_{v}(P)}{v} \sqrt{P_{K}^{X} P_{K}^{Y}}
$$

where $Z_{v}(P)$ is the confidence level associated with the probability $\mathrm{P}$ for a probability distribution function defined by the square root of two $\chi^{2}$ distributions (Grinsted et al., 2004). The relevant codes can be freely downloaded from the website http://www.pol.ac.uk/home/rese-arch/waveletcoherence/.

Corn production shows fluctuating changes in time. The signals of climate variability can explain this variability to a certain extent (Talaee et al., 2014; Huang et al., 2016; Dai et al., 2020). Besides, photosynthesis must be conducted during the growth and development of maize. Therefore, solar activities are expected to have a certain impact on corn yield. Hence, the linkages between maize yield and the signals of climate variability/solar activities at both of the macroscopic and microcosmic scales were explored to fully reveal the impacts of these factors on corn yield in the China mainland, which has the largest population in the world. This work helps to physically reveal the impact of climate change and solar activities on the change in grain production China, thereby being helpful for its reliable maize yield prediction.

\section{Results}

\section{Analysis of the changing periods of detrended maize yield and the IMFs of maize yield in China}

\section{Analysis of the changing periods of detrended maize yield in China}

Lu et al. (2017) found that the all trends of yield of various crops are increasing, mainly caused by technological advances. The trends should be removed before other basic applications are implemented, such as computing the correlation function (Wu et al., 2007). The definition of the residual component in EMD method is almost identical to the definition of the trend when the data span in the trend covers the whole data length (Wu et al., 2007). Based on that, the study gets detrended maize yield data by eliminating the residual of original maize yield data. The continuous wavelet transform (CWT) is used to identify the period of the detrended maize yield data. The results are shown in Fig. 1. Detrended maize yield has a primary period of approximately 18 years, a secondary period of probably 12 years, a third period of roughly 6 years of corn yield and a fourth period of summarily 3 years. It is obviously seen that maize yield fluctuates at different frequencies in time domain (changing from low and medium frequencies to high frequency).

\section{Analysis of the changing periods of the IMFs of maize yield in China}

In this paper, the EMD method was used to decompose the detrended maize yield data. The results were shown in Fig. 2. It can be seen form Fig. 2 that, 1961-2016, detrended maize yield time series in China was decomposed into 4 IMFs and 1 trend item R. Among them, 1) IMF1 reflects a fluctuation of 2 6 years, and the oscillations are more uniform 
throughout the scale period fluctuations. The peaks and valleys of the adjacent years appear one after another, indicating that the short-period maize yield fluctuations are more common, and the maize yield increase and decrease periodicity caused by the scale fluctuations is obvious; 2) IMF2 behaves a fluctuation of $6 \sim 11$ years. The oscillation frequency is slower and the amplitude is relatively stable and small, as well as the fluctuation is relatively flat, which indicates that the maize yield fluctuation intensity of this scale is lighter; 3) there is a 12 19 years fluctuation displayed in IMF3. The period of the oscillation is relatively long and the amplitude is larger than IMF1 and IMF2, indicating that the fluctuation of maize yield at this scale does not occur often, but the intensity is relatively large; 4) it can be seen that IMF4 has a 27 years fluctuation with the long period, large amplitude and intensity indicating that fluctuations in maize yield at this scale rarely occur; 5) for the residual, Maize yield shows an upward trend at 1961-1990, and a downward trend at 1991-2016.

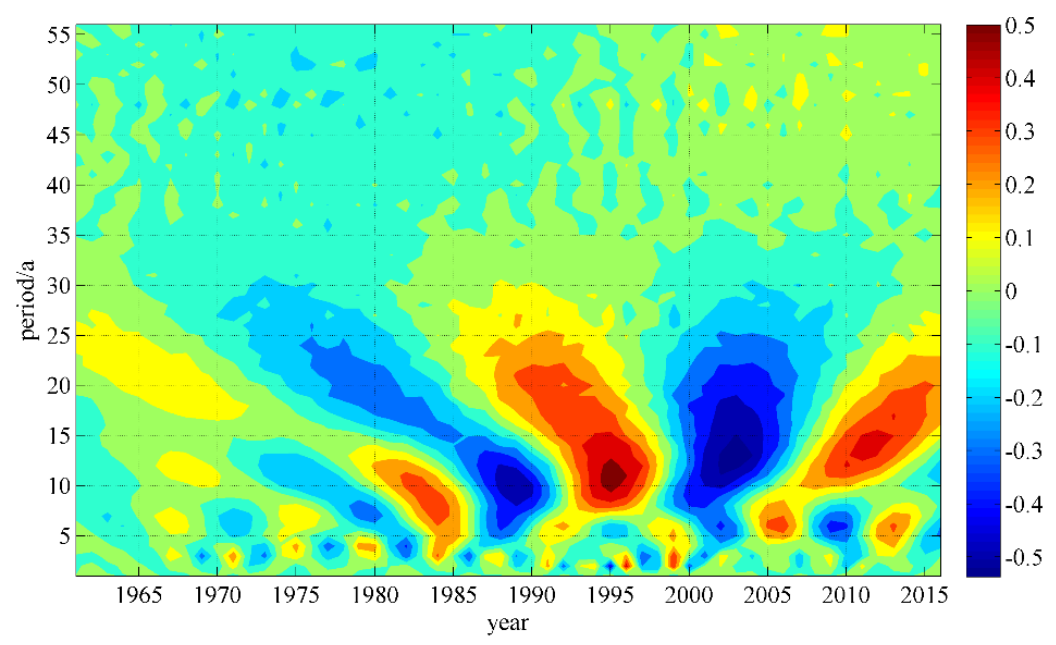

Figure 1. Plot of the continuous wavelet transform of the detrended maize yield data

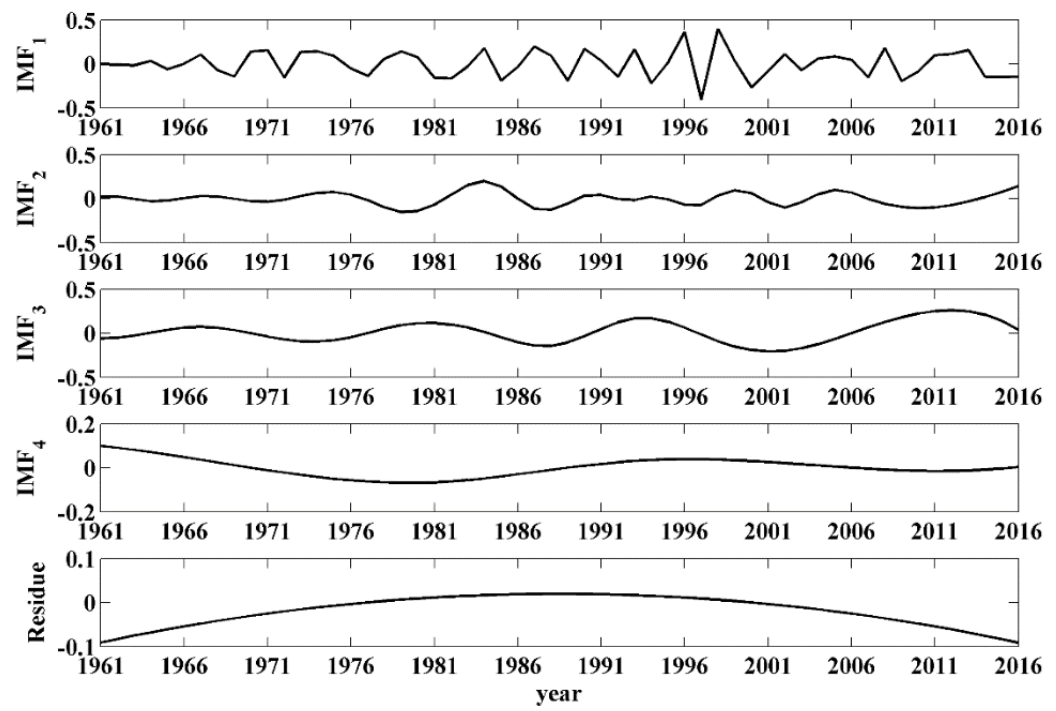

Figure 2. The IMFs of detrended maize yield 


\section{Analysis of the effects of the signals of climate variability and solar activities on detrended maize yield at the macroscopic scale}

As we all know, long-term fluctuations in corn yield are closely related with climatic factors. In this paper, the cross-wavelet analysis was used to analyze the correlations between influencing factors including precipitation, temperature, sunspots, AO, ENSO as well as PDO and detrended maize yield (Fig. 3).
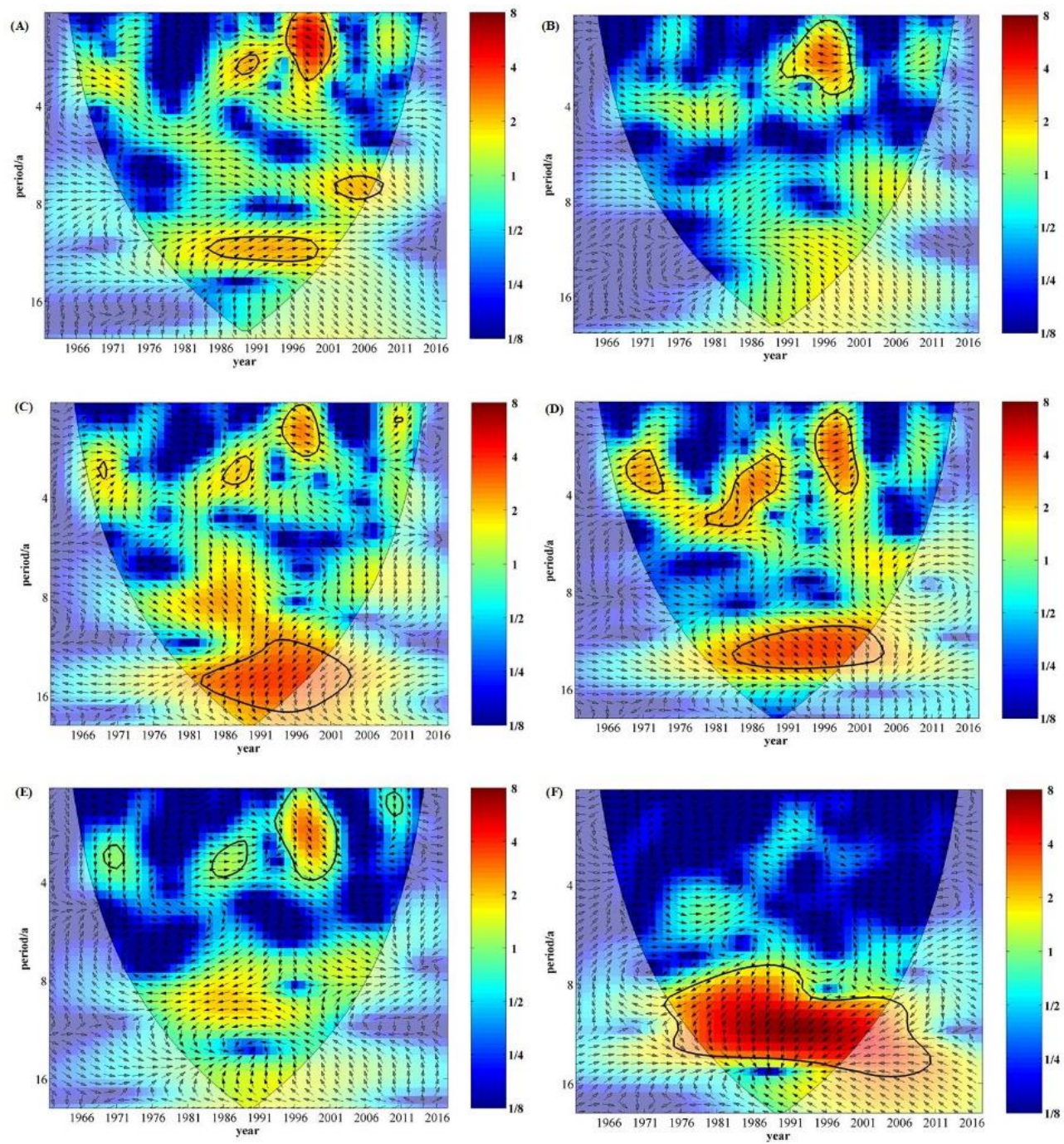

Figure 3. The cross wavelet transforms of signals of climate variability (included precipitation $(A)$, temperature $(B), A O(C), E N S O(D), P D O(E))$ and solar activities (sunspots $(F)$ ) and detrended maize yield, respectively

To reveal the relationship between precipitation and detrended maize yield data in the Chinese mainland, the cross-wavelet analysis was performed and the results were shown in Fig. 3A. Fig. 3A displays that there are statistically positive correlations between precipitation and detrended maize yield with a 1-3 year signal in 1995-2001, a 2.5 year signal in 19871992, a 11 13 year signal in 1984 2000 and a 7.5 year signal in 2002 2008 at the 95\% confidence level. There are some differences in the Fig. 3B. It exhibits the cross wavelet transforms between temperature and detrended maize yield. Temperature has relatively 
significant negative linkages with detrended maize yield at the $95 \%$ confidence level with a 1-3.5 year signal in 1990-2000. These directly demonstrate that precipitation and temperature play an important role in corn yield and have the opposite relationship with corn yield. The results were basically consistent with Zhao et al. (2015b). They found that precipitation and temperature had significant opposite effects on the climate-induced yield of maize from the macroscopic perspective.

AO has comparatively significant positive correlations with detrended maize yield with a 3 year signal in 1967-1970 and a 1.5 year signal in 2009-2011 (Fig. 3C). It also shows negative correlations with detrended maize yield with a 3-3.5 year signal in 1985-1990 and a 1-2.5 year signal in 1994-2000. Similarly, ENSO (Nino3.4) has relatively significant negative linkages with detrended maize yield at the $95 \%$ confidence level with a 3-4 year signal in 1967-1972 and a 1.5-4 year signal in 1995-2001, and it has significant positive correlations with detrended maize yield with a 3-5 year signal in 1980-1990 and a 11 14 year signal in 1982-2004 (Fig. 3D). In addition, Fig. 3E exhibits that PDO has a significant positive correlation with detrended maize yield at the $95 \%$ confidence level with a 1-4 year signal in 1992-2002. It also has a comparatively positive correlation with detrended data at the $95 \%$ confidence level with a 3.5 year signal in 1984-1990. It can be observed from Fig. $3 F$ that sunspots show a statistically significant negative correlation with detrended maize yield at the $95 \%$ confidence level with a 7.5-15 year signal in 1973-2011. In addition, some relevant papers (Kim et al., 2005; Butler et al., 2013; Zhao et al., 2015b) reported that the linkages between maize yield and the signals of climate variability (AO, PDO and ENSO) and solar activities at macroscopic scale, which further verifies the reliability of our findings in this study.

Based on Fig. 3A Fig. $3 F$, it was evident that the signals of climate variability (i.e., precipitation, temperature, ENSO (Nino3.4), AO, PDO) and solar activities (sunspots) had statistically correlations with detrended maize yield. These demonstrate that the short and long-medium periods of maize yield in China are subject to the interaction of above-mentioned factors. It is worth noting that sunspots had the most significant effects on corn yield, and their impacts were concentrated on low frequency regions of maize yield at the macroscopic scale. In contrast, the high-frequency fluctuations were mainly caused by the signals of climate variability at the macroscopic scale. According to influence intensity, this paper found that sunspots had the strongest influence on maize yield, followed by ENSO and $\mathrm{AO}$, followed by precipitation, followed by PDO, and temperature had the weakest influence on maize yield found in Fig. 3.

\section{Analysis of the effects of the signals of climate variability and solar activities on decomposition of detrended maize yield at the microcosmic scale}

Section 3.2 shows that maize yield is the result of a combination of factors. Based on the above analysis, the application of the empirical mode decomposition (EMD) method in this manuscript to decompose maize yield data. Detrended maize yield data was decomposed into 4 intrinsic mode functions (IMF) and a residual component. The cross-wavelet analysis was employed to explore the correlations between the four IMFs and signals of climate variability, which is contributed to a deeper understanding of the impact of the signals of climate variability on maize yield at the microcosmic scale. Similarly, the cross-wavelet analysis was used to analyze the impact of solar activities on the decomposition of detrended maize yield. The results are displayed in Fig. 4 Fig. 9.

As seen from Fig. 4A that there was a statistically positive correlation between precipitation and the IMF1 of corn yield with a 3 year signal in 1987-1992, a 1-3.5 year signal 
in 1995-2001 and a 8 year signal in 2000-2010 at the 95\% confidence level. There were some differences in Fig. 4B compared with Fig. 4A. In the figure, it shows that the cross wavelet transforms between precipitation and the IMF2. Precipitation had statistically significant positive linkages with the IMF2 at the 95\% confidence level with a 4-13 year signal in 19702011. In addition, Fig. $4 C$ displays that precipitation had a comparatively significant positive correlation with the IMF3 at the 95\% confidence level with a 12 year signal in 1977-2002. Fig. $4 \mathrm{D}$ and Fig. $4 \mathrm{E}$ indicated that the correlations between precipitation and IMF4/residue were not significant. These results demonstrated that precipitation mainly impacted the medium-high frequency regions of maize yield and had a certain influence on the low frequency regions.
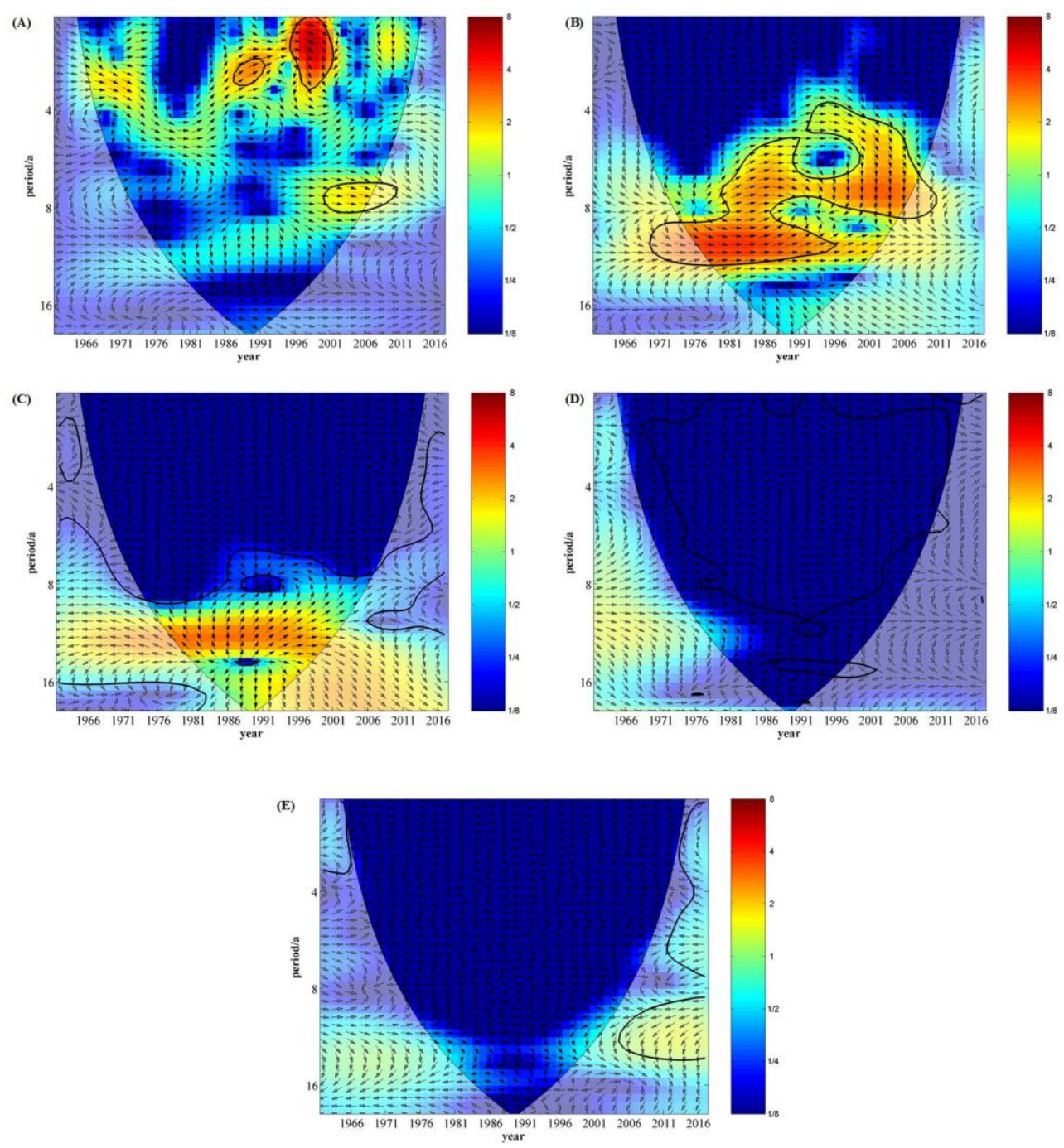

Figure 4. The cross wavelet transforms of precipitation and IMF1 4 and residue of maize yield $(A \sim E)$, respectively

It can be observed from Fig. 5A that temperature showed a statistically significant negative correlation with IMF1 at the $95 \%$ confidence level with a 1.5-3.5 year signal in 1990-2001. There was a positive correlation between precipitation and the IMF1 of corn yield a 4 year signal in 1975-1982 at the 95\% confidence level. There are some differences in Fig. 5B. It reveals that temperature has relatively significant positive 
linkages with the IMF2 at the 95\% confidence level with a 4 year signal in 1992-1997 and a 6 7.5 year signal in 2000-2006. In addition, Fig. 5C displays that temperature has a comparatively significant positive correlation with the IMF3 at the $95 \%$ confidence level with a 13 15 year signal in 1976-2016. Fig. 5D and Fig. 5E have weaker correlations between temperature and IMF4/residue. In a word, temperature concentrated influence on medium and high frequency of corn yield.
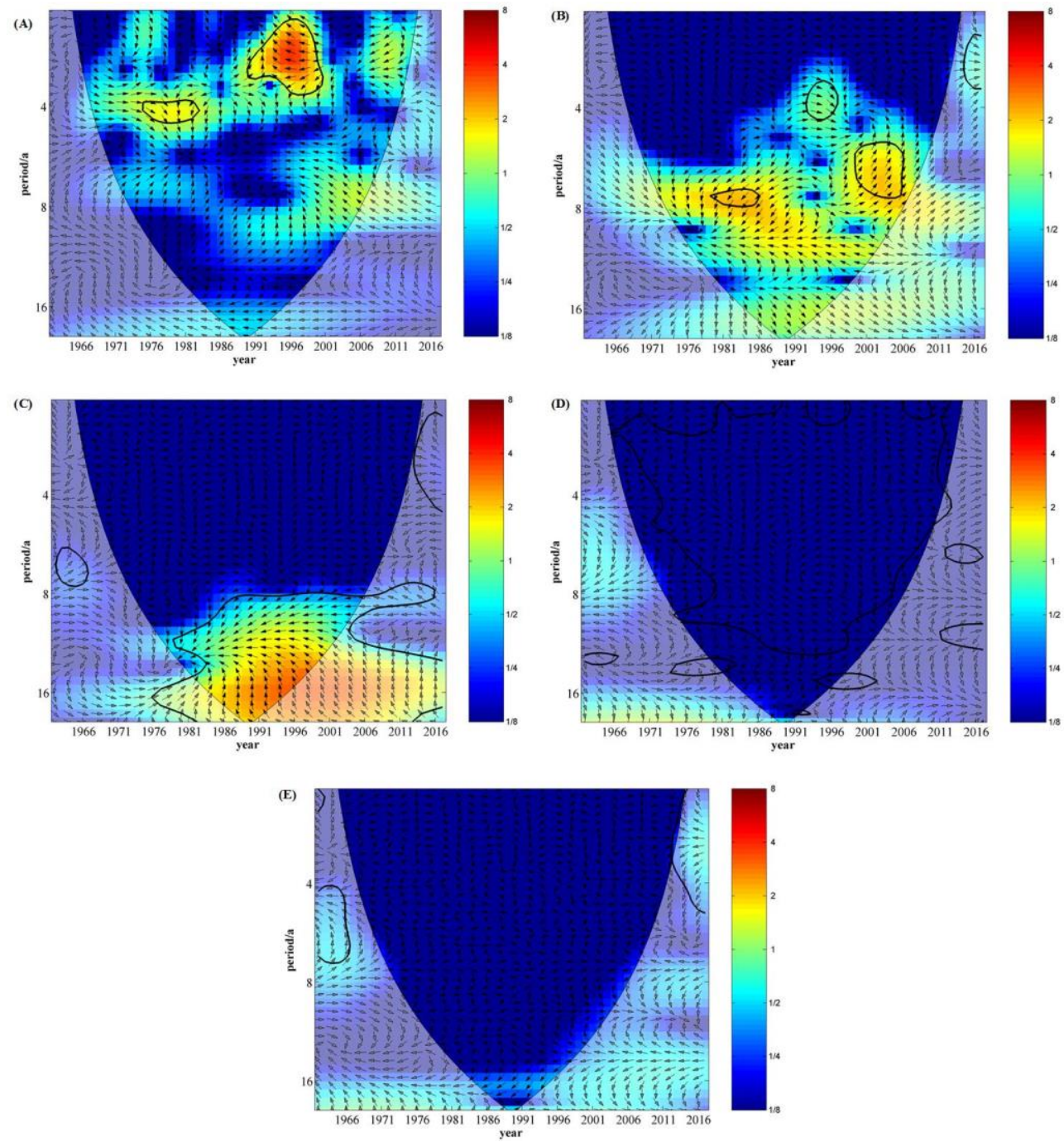

Figure 5. The cross wavelet transforms of temperature and IMF1 4 and residual of maize yield $(A \sim E)$, respectively

Fig. 6A displays that there is a statistically positive correlation between AO and IMF1 of corn yield in the Chinese mainland with a 3-4 year signal in 1967-1972 and it has a significant negative linkages with IMF1 with a 3-4 year signal in 1983-1991 and a 2 year signal in 19941999 at the $95 \%$ confidence level. There are some differences in the Fig. 6B. It exhibits the cross wavelet transforms between AO and IMF2 of corn yield. AO has significant positive linkages with IMF2 at the 95\% confidence level with a 4-10 year signal in 1972-2000 and a significant negative correlation with IMF2 with a 5 year signal (Fig. 6B). It can be observed from Fig. $6 C$ that there is a statistically negative correlation between AO and IMF3 of corn yield with a 9-17 year signal in 1961-2016. No significant relationship between AO and IMF4 
and residual term was found in the Fig. $6 D$ and $6 E$. We can see that AO primarily affects medium and low frequency of maize yield.
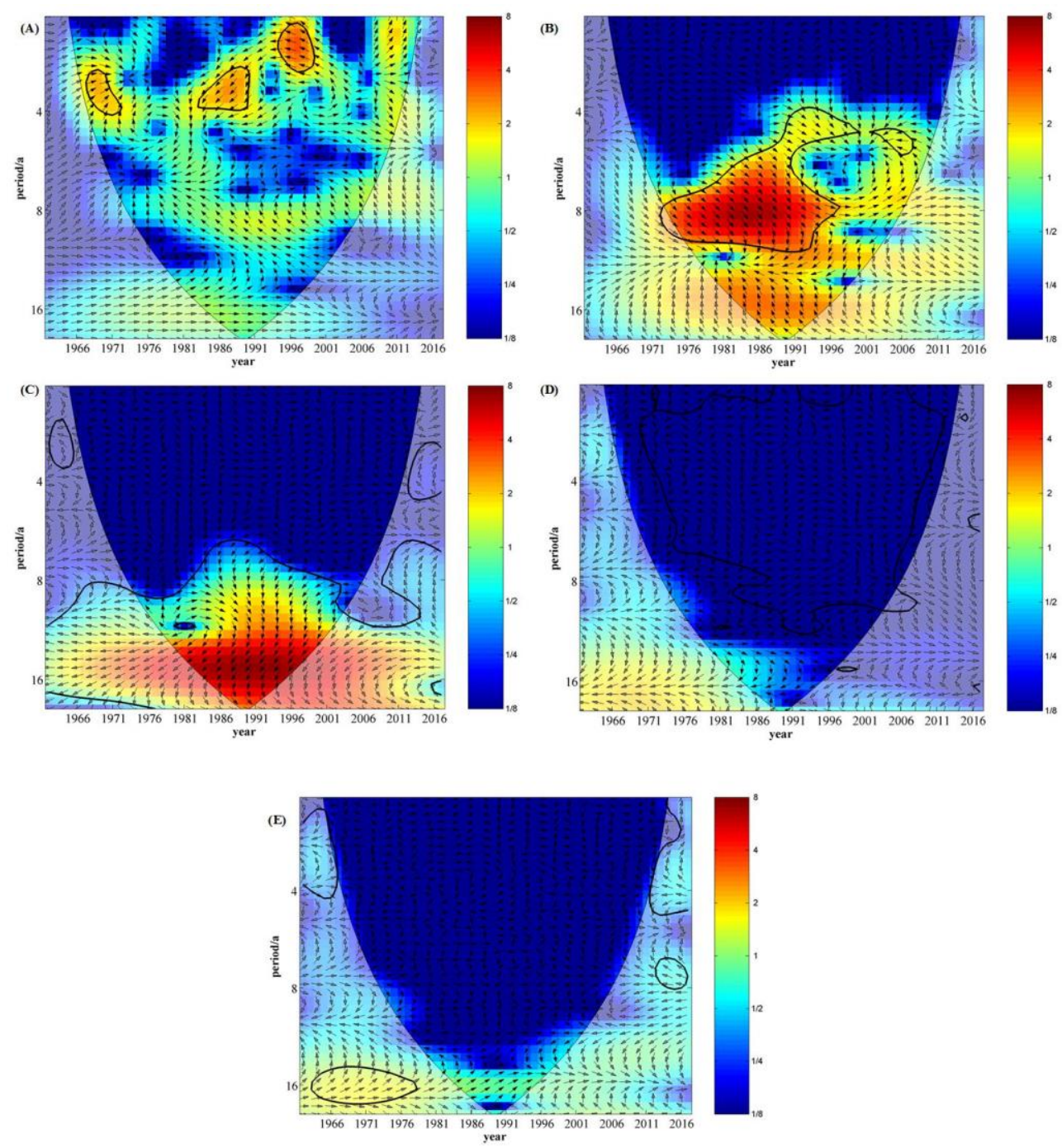

Figure 6. The cross wavelet transforms of $A O$ and IMF1 4 and residue of maize yield (A E), respectively

Similarly, the cross wavelet analysis was applied to reveal the correlations between ENSO (Nino3.4) and the IMFs of maize yield. There are the results shown in Fig. 7 ENSO has a statistically positive correlation with IMF1 of corn yield with a 3-5 year signal in 1968-1991 and a 1.5-4 year signal in 1994-2001 (Fig. 7A). It also shows a significant correlation between ENSO and IMF2 with a 4-7 year in 1982-2007 and a significant positive linkage with IMF2 with a 14 year signal in 1977-2015 (Fig. 7B). Besides, Fig. 7C exhibits a comparatively significant positive correlation with IMF3 at the $95 \%$ confidence level with a 14 year signal in 1976-2016. There are weaker correlations between ENSO and IMF4 and residue seeing from Fig. $7 D$ and $7 E$. Above all, it is obviously seen that ENSO mainly impact on mediumhigh frequency of maize yield. 

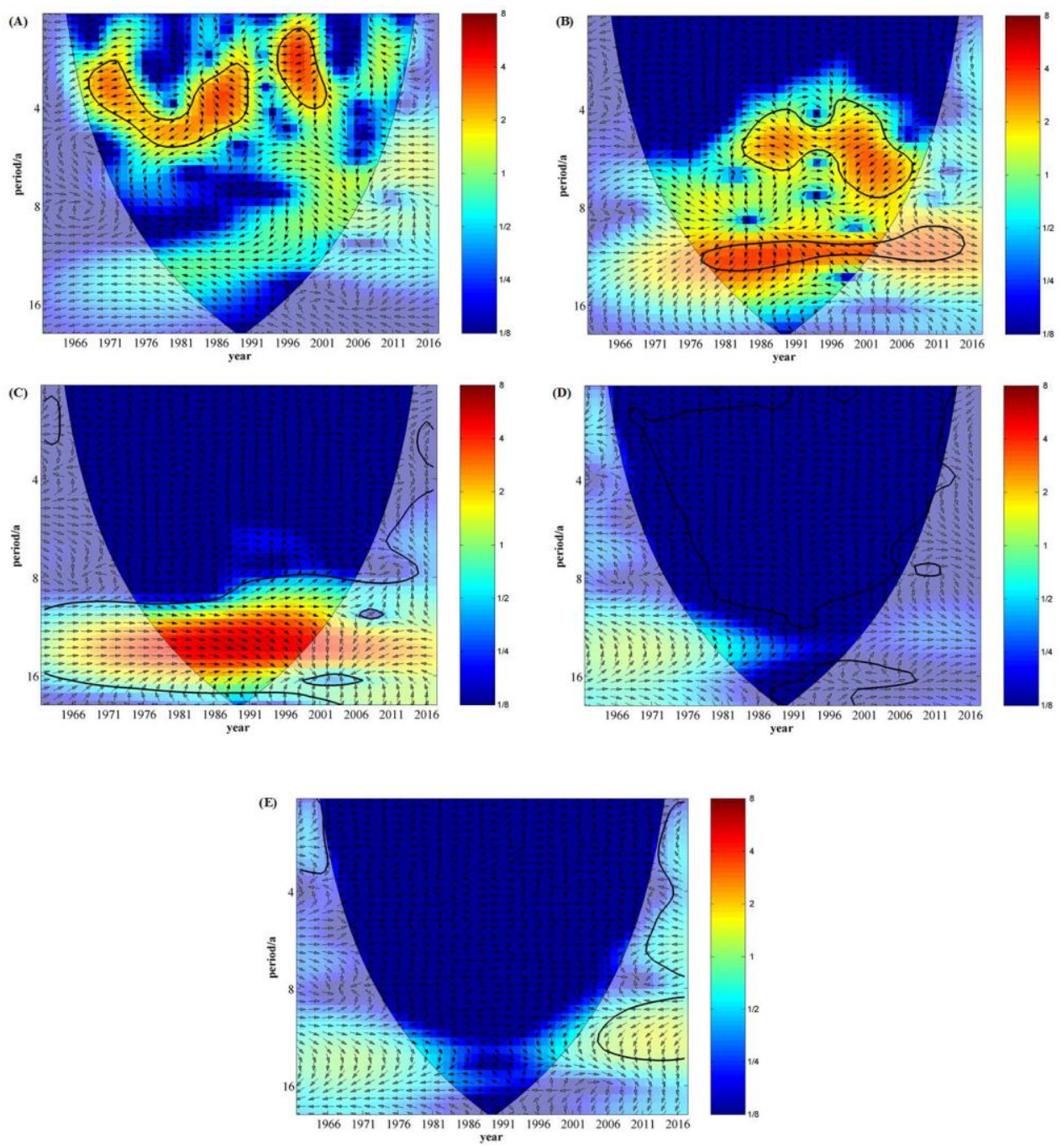

Figure 7. The cross wavelet transforms of ENSO (Nino3.4) and IMF1 4 and residue of maize yield $(A \sim E)$, respectively

In addition, PDO has statistically significant positive linkages with IMF1 of corn yield at the 95\% confidence level with a 1-4 year signal in 1992-2002 and a 3-4 year signal in 1982-1990 (Fig. 8A). It also shows that a comparatively significant correlation between PDO and IMF1 with a 3-4 year signal in 1967-1972. Fig. $8 B$ exhibits PDO has a statistically significant linkage with IMF2 with a 6-9 year signal in 1972-2007. It can be seen from Fig. $8 C$ that PDO has a relatively significant linkage with IMF3 with a 8 16 year signal in 1976-2016. Based on Fig. 8, we can see that PDO mainly influence medium frequency of maize yield.

In addition to analyzing the correlations between signals of climate variability and maize yield components, the cross-wavelet transform was used to explore the linkage the IMFs and solar activities (sunspots). The results are displayed in Fig. 9. It can be seen from Fig. 9A that sunspots have a relatively significant positive linkage with IMF1 of corn yield at the 95\% confidence level with a 4-5 year signal in 1976-1982 and a statistically significant negative linkage with IMF1 with a 8-14 year signal in 1977-2005. Fig. $9 B$ exhibits a statistically significant correlation between sunspots and IMF2 with a 
5-14 year signal in 1966-2016. Sunspots have a comparatively significant linkage with IMF3 with a 8-16 year signal in 1967-2016 (Fig. 9C). Interesting, sunspots mainly influence on medium and low frequency of maize yield components.
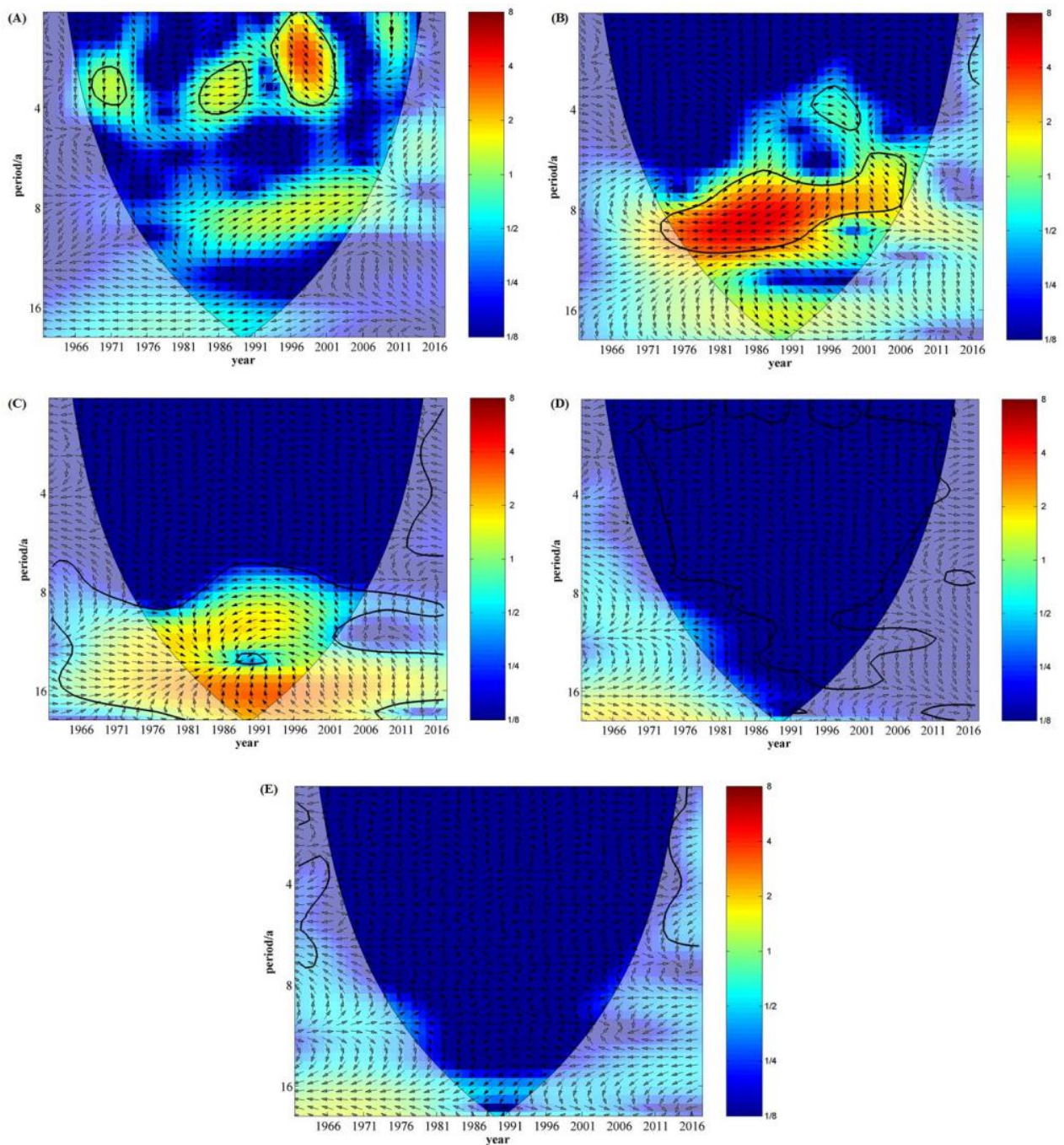

Figure 8. The cross wavelet transforms of PDO and IMF1 4 and residue of maize yield (A E), respectively

Based on Fig. 4 and Fig. 9, it was evident that PDO mainly affect the first and second components of detrended maize yield, and the second and third components of detrended maize yield were mainly impacted by sunspots and $\mathrm{AO}$, whilst the first three components of detrended maize yield were influenced by ENSO and precipitation, moreover, the effect of temperature on detrended maize yield was relatively weak, and mainly affect the first and third component of detrended maize yield. These results indicated that the yield components of corn were not only affected by a single factor, but the result under the combined action of multiple factors, and the influence factors of different components were not the same. As far as was known, there were a number of papers (Plaut et al., 1995; Stonter et al., 2009; Mokhov et al., 2013; Zhao et al., 2015b; Chen et al., 2016) had proved that the signals of climate variability (i.e. precipitation, temperature, ENSO (Nino3.4), AO, PDO) and solar activities 
(sunspots) dominated by different periodic signals, which further verified the rationality and accuracy of the above findings.
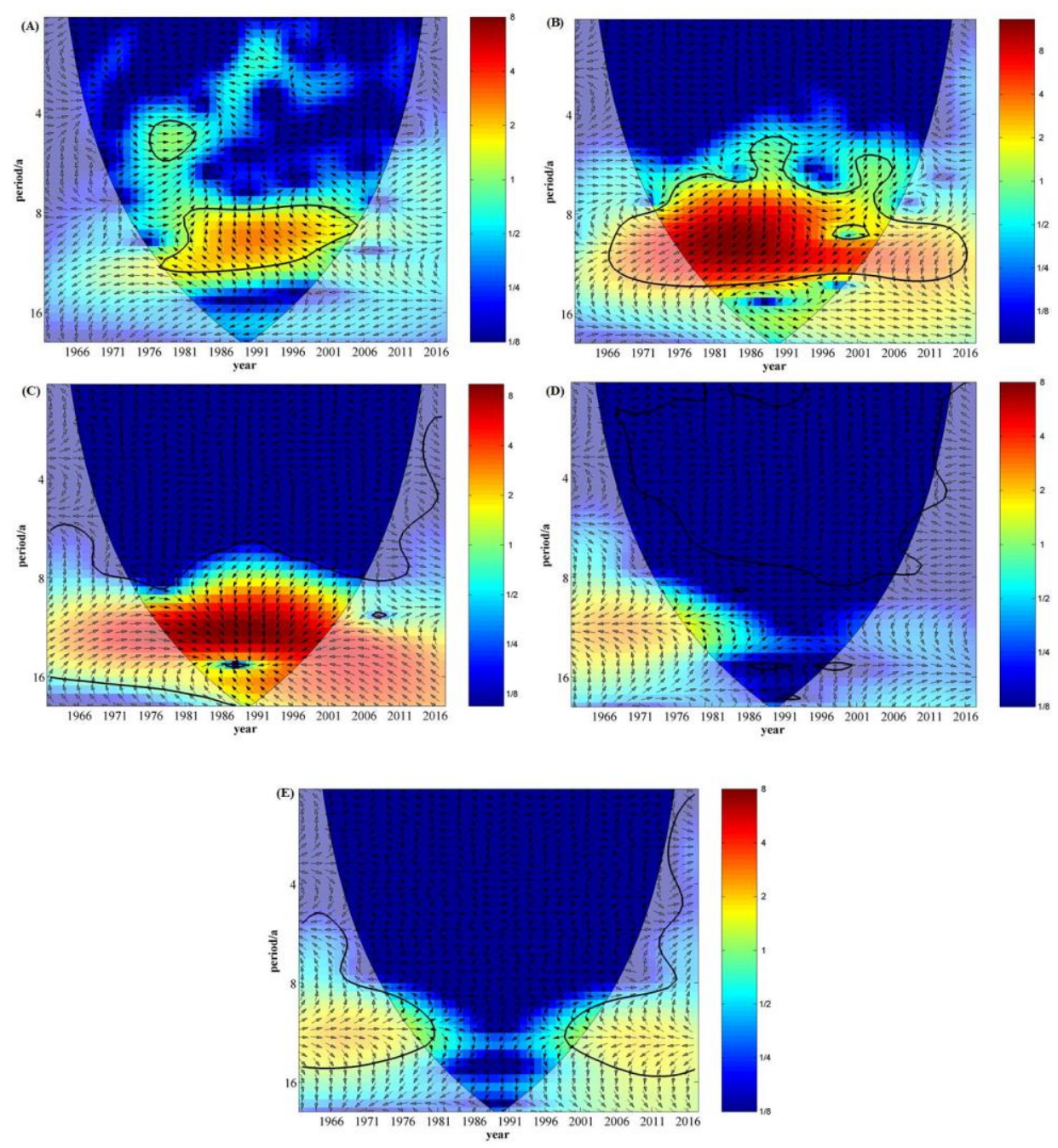

Figure 9. The cross wavelet transforms of sunspots and IMF1 4 and residue of maize yield $(A \sim E)$, respectively

\section{The complete influencing hierarchical framework}

More and more studies indicate that teleconnection factors (e.g. ENSO, AO, PDO) and sunspots have major impact on global atmospheric circulation and regional climatic anomalies (Kenyon et al., 2008; Schubert et al., 2008; Alexander et al., 2009; Dikpati et al., 2010; Owens et al., 2011; Meehl et al., 2013). According to the analysis results in section 3.3, different impacting hierarchical frameworks were constructed for IMF1 3.

It can be seen from Fig. 4A, Fig. 5A, Fig. 7A and Fig. $8 \mathrm{~A}$ that there were statistically positive correlations between precipitation, PDO and the IMF1 of detrended maize yield in 1980-2000, and ENSO had a significant positive linkage with IMF1 of detrended maize yield in 1983-1990. In contrast, temperature and ENSO showed statistically significant negative correlations with IMF1 in 1991-2001. These suggested that precipitation, PDO, ENSO and temperature had significant different effects on the growth of maize, and also suggested that the effect of ENSO on maize yield was different in different years. The effects of aforementioned factors on IMF1 
were extremely complicated and each factor had mutually reinforcing or weakening influence on maize yield. In view of the aforementioned results, the hierarchical framework of ENSO, PDO-climate variability (precipitation, temperature)-the IMF1 of detrended maize yield may be constructed. In other words, there was a chain effect, which was ENSO and PDO definitely showing impact to precipitation/temperature in some degree and in some land areas, and consequently the climate variability in such variables being certainly associated with detrended maize yield.

Compared the correlations in Fig. 4B, Fig. 6B, Fig. 7B, Fig. $8 B$ and Fig. 9B, it can be found that precipitation and teleconnection factors had effect on IMF2 of detrended maize yield in the time domain of sunspots influencing IMF2. Therefore, the framework of sunspotsteleconnection factors (ENSO, AO and PDO)-precipitation-the IMF2 of detrended maize yield linkage may be constructed. In other words, there was a chain effect, which was sunspots definitely showing impact to teleconnection factors in some degree, and subsequently, the teleconnection factors exhibiting impact to precipitation in some degree and in some land areas, and consequently precipitation in such variables being certainly associated with detrended maize yield.

Combine the information of these figures (including Fig. 4C, Fig. 5C, Fig. 6C, Fig. $7 C$ and Fig. $8 \mathrm{C}$ ) to know that the framework of sunspots-teleconnection factors (ENSO, AO)-climatic variability (precipitation, temperature)-the IMF3 of detrended maize yield linkage may be constructed.

Based on that, the cross wavelet method was used to explore the correlation between sunspots and AO, ENSO as well as PDO (Fig. 10) in this study. Fig. 10 showed that sunspots had significant linkages with AO, ENSO and PDO at the 95\% confidence level with an 8 14 year signal in 1966-2001. The findings suggested that sunspots had significant effect on three teleconnection factors, and these also confirmed the first step of the chain effect (for IMF2 and IMF3).
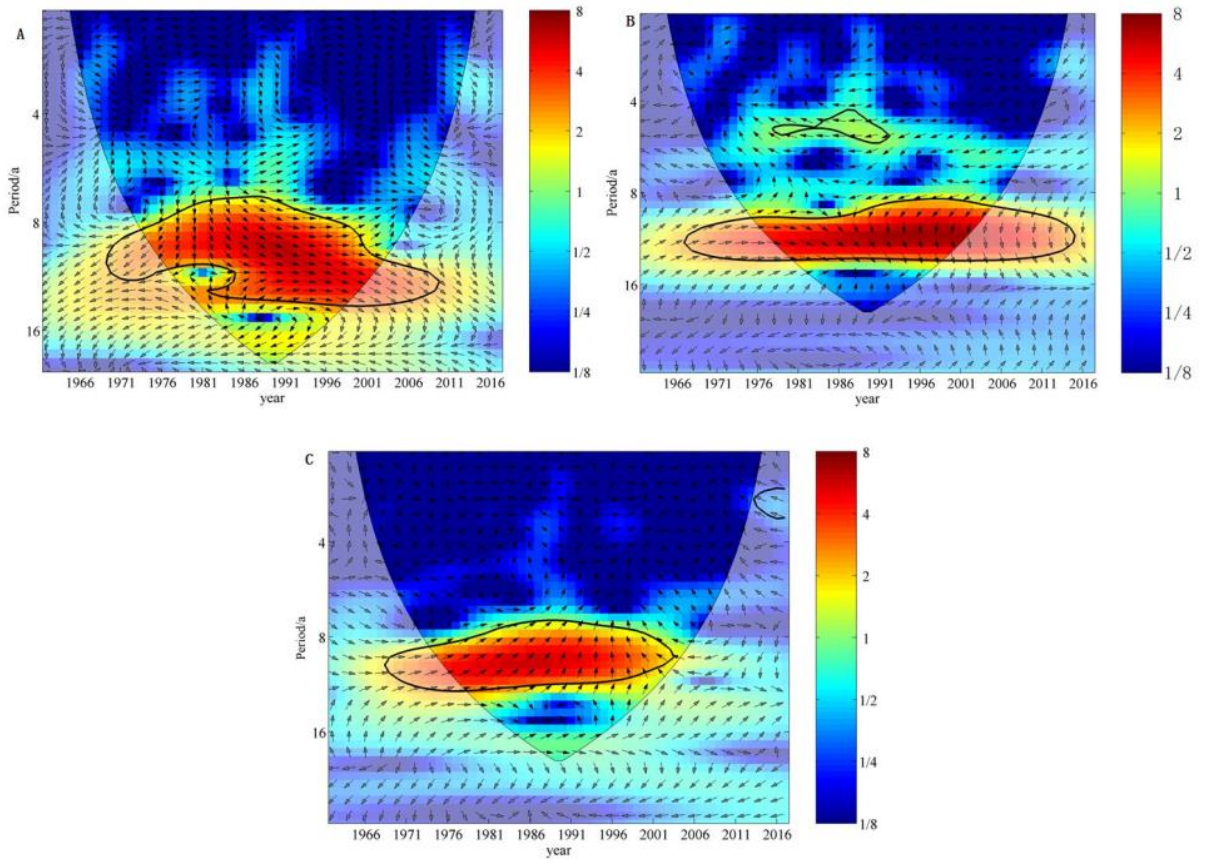

Figure 10. The cross wavelet transforms of sunspots and $A O(A), E N S O(B)$ and $P D O(C)$, respectively 
Both precipitation and temperature were decomposed by means of EMD decomposition in this paper, since they are also affected by various factors. Similar to the Fig. 10, the crosswavelet method was utilized in the study, to explore the relationship between AO, ENSO, PDO and the components of precipitation and temperature (as shown in Fig. 11 and Fig. 12). Fig. 11 displayed that AO and ENSO were significantly correlated with the IMF2 and IMF3 of precipitation, and that there was a significant correlation between PDO and the IMF2 of precipitation. These results indicated that AO, ENSO and PDO had an important influence on precipitation. By contrast, the effects of $\mathrm{AO}$ and ENSO on precipitation were significantly stronger than that of PDO on precipitation. Fig. 12 exhibited that there were significant linkages between AO, ENSO, PDO and the IMF1 and IMF2 of temperature. In comparison, AO and ENSO had a stronger effect on temperature than PDO. In a word, AO, ENSO and PDO had a certain influence on precipitation and temperature. And more, the findings confirmed the first step (for IMF1) and the second step (for IMF2 and IMF3) of the chain effect. As AO, ENSO and PDO had no significant influence on the IMF4 and residual term of precipitation and temperature, the correlations between them are explored in this paper. Three complete influencing hierarchical frameworks were shown in Fig. 13.
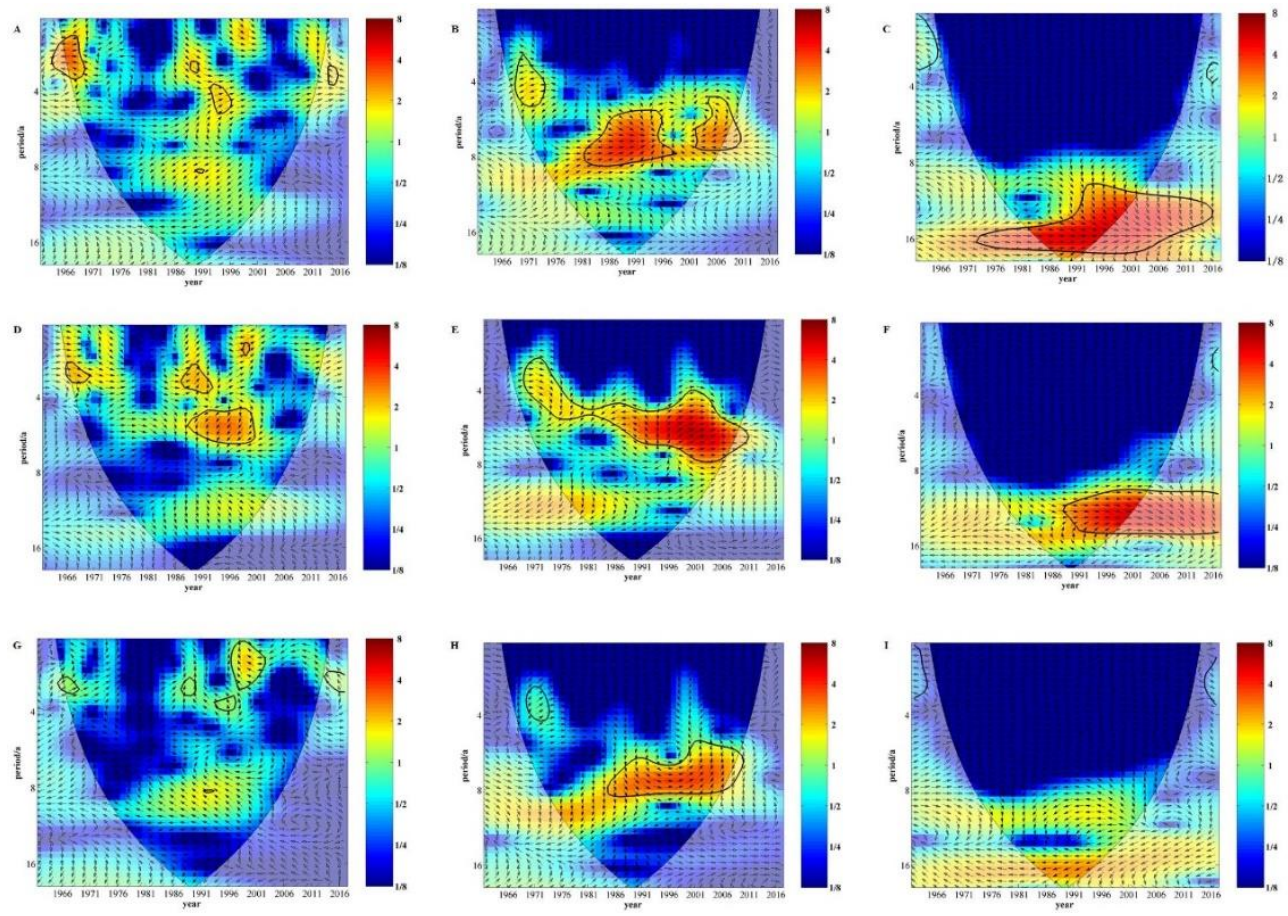

Figure 11. The cross wavelet transforms of $A O$ and the IMF1 (A), IMF2 (B) and IMF3 (C) of precipitation, ENSO and the IMF1 (D), IMF2 (E) and IMF3 (F) of precipitation as well as PDO and the IMF1 $(G), I M F 2(H)$ and IMF3 (I) of precipitation, respectively

Besides the above findings, we can also see from the figure that the signals of climate variability and solar activities have no significant influence on the IMF4 and residual terms of corn yield. This is mainly because the maize yield sequence was detrended in this paper, which made the low-frequency signals in the maize yield sequence be removed, so that the climatic factors had no obvious influence on the detrended maize yield sequence after decomposition. 

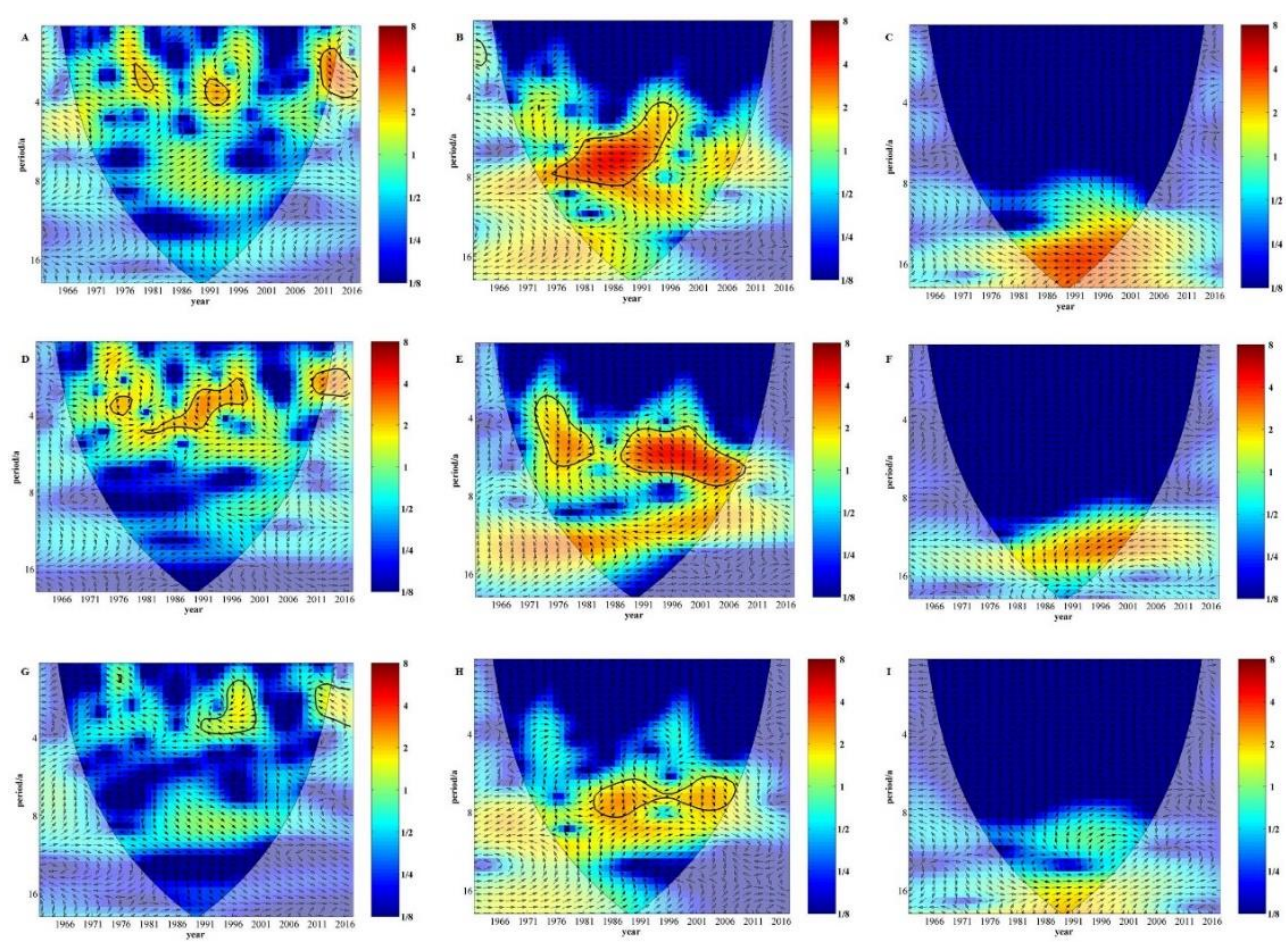

Figure 12. The cross wavelet transforms of AO and the IMF1 (A), IMF2 (B) and IMF3 (C) of temperature, ENSO and the IMF1 (D), IMF2 (E) and IMF3 (F) of temperature as well as PDO and the IMF1 $(G), I M F 2(H)$ and IMF3 (I) of precipitation, respectively
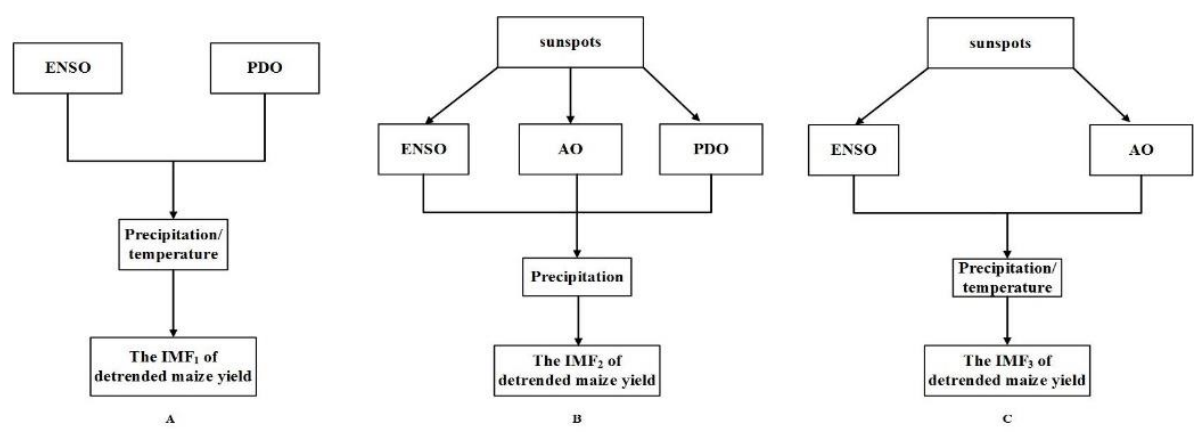

Figure 13. Three complete influencing hierarchical frameworks for IMF1 (A), IMF2 (B) and $\operatorname{IMF3}(C)$

Above all, three frameworks were constructed. These also showed that the effects of signals of climate variability and solar activities on the IMFs of corn yield can not only verify the accuracy of the effects of these factors on maize yield after detrending, but also demonstrate each single component of the IMFs was synthetically affected by the above factors. Based on these findings, this study can provide a new idea for the grain prediction model, that is, the above factors can be used to predict the different components of corn (i.e. ENSO, PDO, precipitation and temperature could be used to predict the IMF1 of maize yield; sunspots, ENSO, AO, PDO and precipitation could be used to predict the IMF2 of maize yield; and sunspots, ENSO, AO, precipitation and temperature could be used to predict the IMF3 of maize yield), and then the predicted results can be combined with relevant means to improve the prediction accuracy of grain yield to a certain extent. 


\section{Discussion}

\section{The necessity of the signals of climate variability and solar activities affect the changes in detrended maize yield at microcosmic scale}

Compared to the effects of precipitation on detrended maize yield and the IMFs of detrended maize yield (Fig. 3A and Fig. 4), it can be discovered that there were statistically positive correlations between precipitation and detrended maize yield with a 1-3 year signal in 1995-2001, a 2.5 year signal in 1987-1992 and a 7.5 year signal in 20022008 at the $95 \%$ confidence level (Fig. 3A), which was basically consistent with the effect of precipitation on detrended maize yield (Fig. 4A). Besides, the interesting findings in Fig. 4B, Fig. 4C and Fig. 3A were that a 11 13 year signal of significance in 1984-2000 (shown in Fig. 3A) was included in the time-frequency domains beyond significance of Fig. $4 \mathrm{~B}$ and Fig. 4C. These findings suggested that precipitation had a significant positive effect on detrended maize yield and mainly affected the fluctuation of detrended maize field in the short and long-medium terms in macroscopic scale, which was fully verified on the microscopic scale (shown in Fig. 4A 4C). Notably, in Fig. 3A, the regions with high energy in the low and medium frequency regions but not exceeding the significance test all exceeded the significance test in Fig. 4B and Fig. 4C. On the one hand, the findings showed that the correlations of precipitation and the IMF1 3 of detrended maize yield were obviously superior to the relationship between precipitation and undecomposed detrended maize yield, suggesting that the microscopic scale to a certain extent, can isolate the influence of other factors; On the other hand, the feature that cannot be expressed on the macroscopic scale was observed on the microscopic scale, which indicated from the side that the yield of maize was subject to the influence of various factors. Similarly, temperature also influenced high frequency of detrended maize yield found from Fig. 3B and Fig. 5A, which was expressed in the time domain in 1990-2001. In contrast, there was a significant negative correlation between temperature and detrended maize yield (compared to Fig. 3A and Fig. 3B). In addition, the discoveries of temperature and detrended maize yield on macroscopic and microscopic scales were basically consistent with that of precipitation and detrended maize yield. In other words, the analyses of the correlations between temperature and the IMF1 3 of detrended maize yield in microscopic scale were the verification and supplement of the relationship between temperature and detrended maize yield.

It can be seen from Fig. $3 C \sim 3 E$ and Fig. 5 8 that the correlations between AO (ENSO, PDO) and detrended maize yield were roughly similar with the relationship between AO (ENSO, PDO) and the IMF1, which demonstrated that AO (ENSO, PDO) had strong effects on the high frequency of detrended maize yield. It was worth noting that sunspots influenced medium frequency of detrended maize yield (a period of $8 \sim 12$ years in the frequency domain) exhibited in Fig. 3F and Fig. 9A, which was different with the effect of the signals of climate variability on detrended maize yield. As we all know, sunspots have an average period of 11 years, which was basically consistent with the frequency domain of sunspots impacting on detrended maize yield. Similar to the comparison of the correlations between precipitation or temperature and detrended maize yield in macroscopic and microscopic scales, the relationship between AO (ENSO, PDO and sunspots) and detrended maize yield had this feature, as well.

Based on the above analyses we all know that the signals of climate variability (including precipitation, temperature, AO, ENSO, PDO) made a difference to high frequency of detrended maize yield. It indicated that the short-term cycle changes of corn 
yield were mainly caused by meteorological factors (which is consistent with the results of Zhao et al. (2013) and Niu et al. (2018)), whilst mid-cycle changes in corn yield were mainly influenced by solar activities. The correlations between the above factors and the components of detrended maize yield proved the correctness of the above conclusion. For this reason, the above factors can be considered as input variables of corn production prediction model to improve the accuracy of the prediction when predicting the future corn production in China. In addition, this study found that not only the cross wavelet analysis between each influence factor and each component of detrended maize yield sequence can verify the relationship between the factors and detrended maize yield on the macroscopic factors and enhance the analysis accuracy, also found at the macroscopic scale some relevant information cannot be accurately captured on the microscopic scale to get the corresponding added. These findings indicate that correlation analysis from the microscopic scale can help reduce information distortion and ensure the accuracy of correlation analysis.

\section{Potential application of the hierarchical frameworks}

Past studies indicated that there were strong relations between solar activities and teleconnection factors (i.e. ENSO, PDO and NAO) (Kapala et al., 1998; Tudhope et al., 2001; Georgieva et al., 2012; Hassan et al., 2016; Maruyama et al., 2017; Liu et al., 2018; Han et al., 2019). Moreover, these teleconnection factors reflect on local weather conditions (precipitation and temperature) (Leathers et al., 1991; Thompson et al., 1998; Higgins et al., 2002; Huang et al., 2017 ; Zhao et al., 2019). In addition, climate variability, especially precipitation and temperature, have been shown to have important effects on crop yields in major crop growing regions in China (Tao, 2012; Wang et al., 2014; Zhang et al., 2015). The research content of the above literature fully shows that the three influencing hierarchical frameworks established in this paper are reasonable. Therefore, it is entirely possible to construct similar influencing hierarchical frameworks for other crops in other areas, such as rice in China, which can provide very reliable basis for food prediction model and reduce the error of food prediction.

\section{Conclusions}

In this study, we examined the effects of the signals of climate variability (i.e., precipitation, temperature, AO, ENSO, PDO) and solar activities (i.e., sunspots) on maize yield in China mainland during the period 1961-2016 at both of the macroscopic and microcosmic scales. First, we got rid of the trend of maize yield data sequence, and the periodical features of the detrended sequence was explored by means of CWT analysis. The detrended maize yield had a primary period of approximately 18 years, a secondary period of probably 12 years, a third period of roughly 6 years of corn yield and a fourth period of summarily 3 years. Then the effects of signals of climate variability and solar activities on detrended maize yield and the IMFs of original maize yield were investigated by cross-wavelet transforms. Findings showed that (1) sunspots had the strongest influence on maize yield, followed by ENSO and AO, followed by precipitation, followed by PDO, and temperature had the weakest influence on maize yield at macroscopic scale; (2) the signals of climate variability mainly impact the high-medium frequency (i.e., frequency domain is 1 4 years and 6 10 years) of maize yield and solar activities primarily influence medium frequency (i.e., frequency domain is $8 \sim 14$ years) for detrended sequence; (3) PDO mainly affect the first and second components of detrended 
maize yield, and the second and third components of detrended maize yield were mainly impacted by sunspots and AO, whilst the first three components of detrended maize yield were influenced by ENSO and precipitation, moreover, the effect of temperature on detrended maize yield was relatively weak, and mainly affect the first and third component of detrended maize yield; (4) the hierarchical framework of ENSO, PDO-climate variability (precipitation, temperature)-detrended maize yield was constructed for IMF1; the hierarchical framework of sunspots-teleconnection factors (ENSO, AO and PDO)-precipitation-detrended maize yield was constructed for IMF2; the hierarchical framework of sunspots-remote correlation factors (ENSO, AO)-climate variability (precipitation, temperature)-detrended maize yield was constructed for IMF3. The results of the present study could contribute to improve the crop prediction (considering signals of climate variability and solar activities as the potential influence factors both on whole and decomposition of the maize yield sequence) and hence would provide possibilities for ensuring food security. This study explored the impact of climate change on maize yield on multi-temporal scales. Therefore, when predicting maize yield based on climatic conditions, it is possible to consider the prediction of grain component resynthesis from a multi-temporal perspective, which may improve the prediction accuracy of maize yield.

Acknowledgements. This research was jointly funded by the National Key Research and Development Program of China (grant number 2017YFC0405900), the Key laboratory research projects of the education department of Shaanxi province (grant number 17JS104), the National Natural Science Foundation of China (grant number 51709221), the Planning Project of Science and Technology of Water Resources of Shaanxi (grant numbers 2015slkj-27 and 2017slkj-19), the China Scholarship Council (grant number 201608610170), the Open Research Fund of State Key Laboratory of Simulation and Regulation of Water Cycle in River Basin (China Institute of Water Resources and Hydropower Research, grant number IWHRSKL-KF201803 and the Doctorate Innovation Funding of Xi'an University of Technology (grant number 310-252071712).

\section{REFERENCES}

[1] Alexander, L. V., Uotila, P., Nicholls, N. (2009): Influence of sea surface temperature variability on global temperature and precipitation extremes. - J. Geophys. Res. Atmos. 114: D18116.

[2] Angstrom, A. (1924): Solar and terrestrial radiation. - Quarterly Journal of the Royal Meteorological Society 50(210): 121-125.

[3] Bai, H., Tao, F., Xiao, D., Liu, F., Zhang, H. (2015): Attribution of yield change for ricewheat rotation system in China to climate change, cultivars and agronomic management in the past three decades. - Climatic Change 135: 539-553.

[4] Battisti, D. S., Naylor, R. L. (2009): Historical warnings of future food insecurity with unprecedented seasonal heat. - Science 323: 240-244.

[5] Butler, E. E., Huybers, P. (2013): Adaptation of US maize to temperature variations. - Nat. Clim. Change 3: 68-72.

[6] Cane, M., Eshel, G., Buckland, R. (1994): Forecasting Zimbabwean maize yield using eastern equatorial Pacific sea surface temperature. - Nature 370: 204-205.

[7] Chen, T., Xia, G., Wilson, L. T., Chen, W., Chi, D. (2016): Trend and Cycle Analysis of Annual and Seasonal Precipitation in Liaoning, China. - Advances in Meteorology 2: 15.

[8] Dai, M., Huang, S., Z., Huang, Q., Leng, G. Y., Guo, Y., Wang, L., Fang, W., Li, P., Zheng, X.D. (2020): Assessing agricultural drought risk and its dynamic evolution characteristics. - Agricultural Water Management 231: 106003. 
[9] Dikpati, M., Gilman, P. A., Kane, R. P. (2010): Length of a minimum as predictor of nest solar cycle's strength. - Geophy. Res. Lett. 37.

[10] Fang, W., Huang, S. Z., Huang, Q., Huang, G., Wang, H., Leng, G., Wang, L., Guo, Y. (2019): Probabilistic assessment of remote sensing-based terrestrial vegetation vulnerability to drought stress of the Loess Plateau in China. - Remote Sensing of Environment 232: 111290.

[11] Garnett, E., Khandekar, M. (1992): The impact of large-scale atmospheric circulations and anomalies on Indian monsoon droughts and floods and on world grain yields: a statistical analysis. - Agricultural and Forest Meteorology 61: 113-128.

[12] Georgieva, K., Kirov, B., Koucká Knížová, P., Mosna, Z., Kouba, D., Asenovska, Y. (2012): Solar influences on atmospheric circulation. - Journal of Atmospheric and SolarTerrestrial Physics 90-91(1): 15-25.

[13] Godfray, H. C. J., Beddington, J. R., Crute, I. R., Haddad, L., Lawrence, D., Muir, J. F., Pretty, J., Robinson, S., Thomas, S. M., Toulmin, C. (2010): Food security: the challenge of feeding 9 billion people. - Science 327: 812-818.

[14] Grinsted, A., Moore, J. C., Jevrejeva, S. (2004): Application of the cross wavelet transform and wavelet coherence to geophysical time series. - Nonlinear Proc. Geoph. 11: 561-566.

[15] Guo, Y., Huang, S. Z., Huang, Q., Leng, G. Y., Fang, W., Wang, L., Wang, H. (2020): Propagation thresholds of meteorological drought for triggering hydrological drought at various levels. - Sci. Total Environ. 712: 136502.

[16] Hammer, G. L., Hansen, J. W., Phillips, J. G., Mjelde, J. W., Hill, H., Love, A., Potgieter, A. (2001): Advances in application of climate prediction in agriculture. - Agricultural Systems 70: 515-553.

[17] Han, Z. M., Huang, S. Z., Huang, Q., Leng, G. Y., Wang, H., He, L., Fang, W., Li, P. (2019): Assessing GRACE-based terrestrial water storage anomalies dynamics at multitimescales and their correlations with teleconnection factors in Yunnan Province, China.Journal of Hydrology 574: 836-850. DOI: 10.1016/j.jhydrol.2019.04.093.

[18] Hanjra, M. A., Qureshi, M. E. (2010): Global water crisis and future food security in an era of climate change. - Food Policy 35(5): 365-377.

[19] Hassan, D., Iqbal, A., Hassan, S. A., Abbas, S., Ansari, M. R. K. (2016): Sunspots and ENSO relationship using Markov method. - Journal of Atmospheric and Solar-Terrestrial Physics 137: 53-57.

[20] He, Q., Zhou, G., Lü, X., Zhou, M. (2019): Climatic suitability and spatial distribution for summer maize cultivation in China at 1.5 and $2.0^{\circ} \mathrm{C}$ global warming. - Science Bulletin 64(10): 690-697.

[21] Higgins, R. W., Leetmaa, A., Kousky, V. E. (2002): Relationships between climate variability and winter temperature extremes in the United States. - Journal of Climate 15(13): 1555-1572.

[22] Huang, N. E., Shen, Z., Long, S. R., Wu, M. L. C., Shih, H. H., Zheng, Q., Yen, N. C., Tung, C.-C., Liu, H. H. (1998): The empirical mode decomposition and the Hilbert spectrum for nonlinear and non-stationary time series analysis. - Proceedings of the Royal Society of London A: Mathematical, physical and engineering sciences 454: 903-995.

[23] Huang, N. E., Wu, M.-L. C., Long, S. R., Shen, S. S. P., Qu, W. D., Gloersen, P., Fan, K. L. (2003): A confidence limit for the empirical mode decomposition and Hilbert spectral analysis. - Proceedings of the Royal Society of London A: Mathematical, physical and engineering sciences 459: 2317-2345.

[24] Huang, S. Z., Huang, Q., Chang, J. X., Leng, G. Y. (2016): Linkages between hydrological drought, climate indices and human activities: a case study in the Columbia River Basin. Int. J. Climatol. 36(1): 280-290.

[25] Huang, S. Z., Li, P., Huang, Q., Leng, G. Y., Hou, B. B., Ma, L. (2017): The propagation from meteorological to hydrological drought and its potential influence factors. - Journal of Hydrology 547: 184-195. 
[26] Huang, S. Z., Zheng, X. D., Ma, L., Wang, H., Huang, Q., Leng, G. Y., Meng, E., Guo, Y. (2020): Quantitative contribution of climate change and human activities to vegetation cover variations based on GA-SVM model. - Journal of Hydrology 584: 124687.

[27] Hudgins, L., Friehe, C. A., Mayer, M. E. (1993): Wavelet transforms and atmospheric turbulence. - Phys. Rev. Lett. 71: 3279-3282.

[28] Hunt, L. A., Kuchar, L., Swanton, C. J. (1998): Estimation of solar radiation for use in crop modeling. - Agricultural and Forest Meteorology 91(3/4): 293-300.

[29] IPCC. (2014): Climate Change 2014: Synthesis Report. - In: Pachauri, R. K., Meyer, L. A. (eds.) Core Writing Team, Contribution of Working Groups I, II and III to the Fifth Assessment Report of the Intergovernmental Panel on Climate Change. IPCC, Geneva, Switzerland (151 pp).

[30] Kane, R. P. (2008): Prediction of solar cycle 24 based on the Gnevyshev-Ohl-Kopecky rule and the three-cycle periodicity scheme. - Annales Geophysicae 26(11): 3329-3339.

[31] Kapala, A., Mächel, H., Flohn, H. (1998): Behaviour of the centres of action above the Atlantic since 1881. Part II: Associations with regional climate anomalies. - Int. J. Climatol 18(1): 23-36.

[32] Kenyon, J., Hegerl, G. C. (2008): Influence of modes of climate variability on global temperature extremes. - J. Clim. 21: 3872-3889.

[33] Kim, M., McCarl, B. A. (2005): The agricultural value of information on the North Atlantic oscillation: yield and economic effects. - Clim. Change 71: 117-139.

[34] Knox, J., Hess, T., Daccache, A., Wheeler, T. (2012): Climate change impacts on crop productivity in Africa and South Asia. - Environ. Res. Lett. 7(3): 034032.

[35] Kummu, M., de Moel, H., Porkka, M., Siebert, S., Varis, O., Ward, P. J. (2012): Lost food, wasted resources: global food supply chain losses and their impacts on freshwater, cropland, and fertiliser use. - Sci. Total Environ. 438: 477-489.

[36] Lansigan, F. P., de los Santos, W. L., Coladilla, J. O. (2000): Agronomic impacts of climate variability on rice production in the Philippines. - Agric. Ecosyst. Environ. 82(1-3): 129137.

[37] Leathers, D. J., Yarnal, B., Palecki, M. A. (1991): The Pacific/North American teleconnection pattern and United States climate. Part I: Regional temperature and precipitation associations. - J. Clim. 4(5): 517-528.

[38] Liu, S. Y., Huang, S. Z., Xie, Y. Y., Leng, G. Y., Huang, Q., Wang, L., Xue, Q. (2018): Spatial-temporal changes of rainfall erosivity in the loess plateau, China: Changing patterns, causes and implications. - Catena 166: 279-289.

[39] Li, Z. Y., Huang, S. Z., Liu, D. F., Leng, G. Y., Zhou, S., Huang, Q. (2020): Assessing the effects of climate change and human activities on runoff variations from a seasonal perspective. Stochastic Environmental Research and Risk Assessment, in press. DOI: 10.1007/s00477-020-01785-1

[40] Lobell, D. B., Burke, M. B., Tebaldi, C., Mastrandrea, M. D., Falcon, W. P., Naylor, R. L. (2008): Prioritizing climate change adaptation needs for food security in 2030. - Science 319(5863): 607-610.

[41] Lu, J., Carbone, G. J., Gao, P. (2017): Detrending crop yield data for spatial visualization of drought impacts in the United States, 1895-2014. - Agricultural \& Forest Meteorology 237-238: 196-208.

[42] Magrin, G. O., Travasso, M. I., Rodríguez, G. R. (2005): Changes in climate and crop production during the 20th century in Argentina. - Clim. Change 72: 229-249.

[43] Mantua, N. J., Hare, S. R., Zhang, Y., Wallace, J. M., Francis, R. C. (1997): A Pacific interdecadal climate oscillation with impacts on salmon production. - Bull. Amer. Meteor. Soc. 78: 1069-1079.

[44] Maruyama, F., Kai, K., Morimoto, H. (2017): Wavelet-based multifractal analysis on a time series of solar activity and PDO climate index. - Advances in Space Research 60(6): 1363-72. 
[45] Meehl, G. A., Arblaster, J. M., Marsh, D. R. (2013): Could a future IGrand Solar Minimum like the Maunder Minimum stop global warming? - Geophy. Res. Lett. 40.

[46] Mehta, V. M., Rosenberg, N. J., Mendoza, K. (2012): Simulated impacts of three decadal climate variability phenomena on dryland corn and wheat yields in the Missouri River Basin. - Agricultural \& Forest Meteorology 152(1): 109-124.

[47] Mokhov, I. I., Eliseev, A. V. (2013): Temperature Patterns. - Reference Module in Earth Systems and Environmental Sciences.

[48] Naylor, R. L., Falcon, W. P., Rochberg, D., Wada, N. (2001): Using El Nino/Southern Oscillation climate data to predict rice production in Indonesia. - Climatic Change 50: 255265.

[49] Nelson, G. C., Rosegrant, M. W., Koo, J., Robertson, R., Sulser, T., Zhu, T., Ringler, C., Msangi, S., Palazzo, A., Batka, M., Magalhaes, M., Valmonte-Santos, R., Ewing, M., Lee, D. (2009): Climate Change Impacts on Agriculture and Costs of Adaptation (Research report). - International Food Policy Research Institute, Washington, DC.

[50] Nigam, S., Barlow, M., Berbery, E. H. (1999): Analysis links Pacific decadal variability to drought and streamflow in United States. - EOS Trans. Amer. Geophys. Union 80(51): 621.

[51] Niu, J., Liu, Q., Kang, S., Zhang, X. (2018): The response of crop water productivity to climatic variation in the upper-middle reaches of the Heihe River basin, Northwest China. - Journal of Hydrology 563: 909-926.

[52] Owens, M. J., Lockwood, M., Barnard, L., Davis, C. J. (2011): Solar cycle 24: Implications for energetic particles and long-term space climate change. - Geophy. Res. Lett. 38.

[53] Phillips, J. G., Cane, M. A., Rosenzweig, C. (1998): ENSO, seasonal rainfall patterns and simulated maize yield variability in Zimbabwe. - Agric. For. Meteorol. 90: 39-50.

[54] Planting Industry Management Department, Ministry of Agriculture and Rural Affairs of the People's Republic of China (2018): http://202.127.42.157/mo-azzys/nongqing.aspx.

[55] Plaut, G., Ghil, M., Vautard, R. (1995): Interannual and interdecadal variability in 335 years of Central England temperatures. - Science 350: 324-327.

[56] Podestá, G., Letson, D., Messina, C., Royce, F., Ferreyra, R. A., Jones, J., Hansen, J., Llovet, I., Grondona, M., O’Brien, J. J. (2002): Use of ENSO-related climate information in agricultural decision making in Argentina: a pilot experience. - Agricultural Systems 74(3): 371-392.

[57] Ren, K., Huang, S. Z., Huang, Q., Wang, H., Leng, G.Y., Fang, W., Li, P. (2019): Assessing the reliability, resilience, and vulnerability of water supply system under multiple uncertain sources. - Journal of Cleaner Production 252: 119806.

[58] Ringler, C. (2008): Climate Variability and Change Impact on Water and Food Outcomes. - International Food Policy Research Institute (IFPRI), Washington, DC.

[59] Rosenzweig, C., Iglesias, A., Yang, X. B., Epstein, P. R., Chivian, E. (2001): Climate Change and Extreme Weather Events; Implications for Food Production, Plant Diseases, and Pests. - Global Change and Human Health 2(2): 90-104.

[60] Schubert, S. D., Chang, Y., Suarez, M. J., Pegion, P. J. (2008): ENSO and wintertime exreme precipitaton events over the contiguous United States. - J. Clim. 21: 22-39.

[61] Smith, P. (2013): Delivering food security without increasing pressure on land. - Glob. Food Sec. 2(1): 18-23.

[62] Stoner, A. M. K., Hayhoe, K., Wuebbles, D. J. (2009): Assessing general circulation model simulations of atmospheric teleconnection patterns. - Journal of Climate 22(22): 43484372.

[63] Talaee, P. H., Tabari, H., Ardakani, S. S. (2014): Hydrological drought in the west of Iran and possible association with large-scale atmospheric circulation patterns. - Hydrol. Process. 28: 764-773.

[64] Tao, F. L. (2012): Response of crop yields to climate trends since 1980 in China. - Clim. Res. 54: 233-247. 
[65] Thompson, D. W. J., Wallace, J. M. (1998): The Arctic oscillation signature in the wintertime geopotential height and temperature fields. - Geophys. Res. Lett. 25(9): 12971300.

[66] Tian, L., Li, J., Bi, W., Zuo, S., Li, L., Li, W., Sun, L. (2019): Effects of waterlogging stress at different growth stages on the photosynthetic characteristics and grain yield of spring maize (Zea mays L.) Under field conditions. - Agricultural Water Management 218: 250258.

[67] Torrence, C., Compo, G. P. (1998): A practical guide to wavelet analysis. - Bull. Am. Meteorol. Soc. 79(1): 61-78.

[68] Tudhope, A. W., Chilcott, C. P., McCulloch, M. T., Cook, E. R., Chappell, J., Ellam, R. M., Lea, D. W., Lough, J. M., Shimmield, G. B. (2001): Variability in the El Niño-Southern Oscillation through a glacial interglacial cycle. - Science 291(5508): 1511-1517.

[69] Walker, G. T. (1923): Correlation in seasonal variations of weather. VIII. A preliminary study of world-weather. - Mem. Indian Meteor. Dep. 24(Part 4): 75-131.

[70] Wang, P., Zhang, Z., Song, X., Chen, Y., Wei, X., Shi, P., Tao, F. (2014): Temperature variations and rice yields in China: historical contributions and future trends. - Clim. Change 124: 777-789.

[71] Wu, Z., Huang, N. E., Long, S. R., Peng, C.-K. (2007): On the trend, detrending, and variability of nonlinear and nonstationary time series. - Proc. Natl. Acad. Sci. U.S.A. 104(38): 14889-14894.

[72] Xu, S., Yu, Z., Zhang, K., Ji, X., Yang, C., Sudicky, E. A. (2018): Simulating canopy conductance of the, Haloxylon ammodendron, shrubland in an arid inland river basin of northwest China. - Agricultural and Forest Meteorology 249: 22-34.

[73] You, L., Rosegrant, M. W., Wood, S., Sun, D. (2009): Impact of growing season temperature on wheat productivity in China. - Agric. For. Meteorol. 149: 1009-1014.

[74] Yu, Y. H., Zhang, H. B., Singh, V. P. (2018): Forward Prediction of Runoff Data in DataScarce Basins with an Improved Ensemble Empirical Mode Decomposition (EEMD) Model. - Water 10(4): 388.

[75] Zhang, Y., Wallace, J. M., Battisti, D. S. (1997): ENSO-like interdecadal variability: 1900-93. - J. Climate 10: 1004-1020.

[76] Zhang, T. Y., Zhu, J., Yang, X. G., Zhang, X. Y. (2008): Correlation changes between rice yields in North and Northwest China and ENSO from 1960 to 2004. - Agricultural and forest meteorology 148(6-7): 1021-1033.

[77] Zhang, Z., Song, X., Tao, F. L., Zhang, S., Shi, W. J. (2015): Climate trends and crop production in China at county scale, 1980 to 2008. - Theor. Appl. Climatol. 123: 291-302.

[78] Zhao, J., Wang, H. (2013): Development process, problem and countermeasure of maize production in China. - J. Agric. Sci. Technol. 15(3): 1-6.

[79] Zhao, J. F., Guo, J. P., Mu, J. (2015a): Exploring the relationships between climatic variables and climate-induced yield of spring maize in Northeast China. - Agric. Ecosyst. Environ. 207: 79-90.

[80] Zhao, J. F., Guo, J. P., Xu, Y. H., Mu, J. (2015b): Effects of climate change on cultivation patterns of spring maize and its climatic suitability in Northeast China. - Agric. Ecosyst. Environ. 202: 178-187.

[81] Zhao, X. H., Chen, X., Xu, Y. X., Xi, D. J., Zhang, Y. B., Zheng, X. Q. (2017): An EMDBased Chaotic Least Squares Support Vector Machine Hybrid Model for Annual Runoff Forecasting. - Water 9(3): 153.

[82] Zhao, J., Huang, S. Z., Huang, Q., Wang, H. Leng, G. Y., Peng, J., Dong, H. X. (2019): Copula-Based Abrupt Variations Detection in the Relationship of Seasonal VegetationClimate in the Jing River Basin, China. - Remote Sensing 11: 1628.

[83] Zhao, J., Huang, S. Z., Huang, Q., Leng, G. Y., Wang H., Li, P. (2020a): Watershed waterenergy balance dynamics and their association with diverse influencing factors at multiple time scales. - Sci. Total Environ. 711: 135189. 
[84] Zhao, J., Huang, S. Z., Huang, Q., Wang, H., Leng, G. Y., Fang, W. (2020b): Time-lagged response of vegetation dynamics to climatic and teleconnection factors. - Catena 189: 104474. 